\title{
A Case Report of Alendronate Treatment over 20 Years on Osteoporosis and Literature Review
}

\author{
Jinmei Deng1 ${ }^{*}$, Enjing Chen ${ }^{1 *}$, Suming Jie ${ }^{1}$, Xiuzhen Cai ${ }^{1}$, Dongmei Cai ${ }^{2 \#}$ \\ ${ }^{1}$ Outpatient Clinic of Internal Medicine Department, The First Affiliated Hospital, Sun Yat-sen University, Guangzhou, China \\ ${ }^{2}$ Department of Osteoporosis, The First Affiliated Hospital, Sun Yat-sen University, Guangzhou, China \\ Email: ${ }^{* 13500037898 @ 163 . c o m ~}$
}

How to cite this paper: Deng, J.M., Chen, E.J., Jie, S.M., Cai, X.Z. and Cai, D.M. (2018) A Case Report of Alendronate Treatment over 20 Years on Osteoporosis and Literature Review. Case Reports in Clinical Medicine, 7, 430-440.

https://doi.org/10.4236/crcm.2018.76037

Received: May 21, 2018

Accepted: June 19, 2018

Published: June 22, 2018

Copyright (c) 2018 by authors and Scientific Research Publishing Inc. This work is licensed under the Creative Commons Attribution International License (CC BY 4.0).

http://creativecommons.org/licenses/by/4.0/

\section{Open Access}

\begin{abstract}
Osteoporosis is commonly seen in aged people, but not much attention is paid to it. Patient compliance is challenged by many factors, including long-time treatment and high rates of fatality and disability caused by fragility fractures. With age-related changes, the treatment will last for a lifetime. A clinical case of postmenopausal patient who had received incontinuous treatment of alendronate for 20 years was studied in this article. As the level of compliance varied in different treatment phases, the curative outcome of this patient was altered. This study also presented a literature review to discuss the current situation, treatment and compliance of osteoporosis in China and the corresponding influences on bone mineral density (BMD) and prognosis. Hopefully, this study can increase physicians' awareness of osteoporosis in clinical treatment and its pharmacotherapy and treatment course.
\end{abstract}

\section{Keywords}

Osteoporosis, Alendronate, BMD, Treatment Course, Compliance, Prognosis

\section{Introduction}

Osteoporosis (OP) is commonly seen in the middle-aged and elderly population in China, which is caused by a variety of factors, characterized by a decrease in bone mass and a deterioration in bone microarchitecture that can lead to a greater risk of fracture and fracture-prone metabolic bone diseases (MBDs). The clinical manifestations of OP mainly include pain, fracture, humpback and height loss [1]. As China's aging population grows, there are an increasing ${ }^{\star}$ Authors contributed equally. 
number of patients diagnosed with OP since it is an age-related disease that can lead to fragility fractures with high disabling and fatality rates. Owing to the concerns from national health organizations and the heightened awareness among patients, the treatment for OP has received significant attention. OP treatment can be basically divided into two categories, namely inhibition of bone resorption and acceleration of bone formation. Bisphosphonates are the most widely used bone resorption inhibitors, especially alendronate for clinical application. However, considering that patients with OP usually have hidden symptoms and poor compliance to treatments due to varied drugs treatment for all kinds of chronic diseases. In absence of discomfort and considering the long treatment course, patients with OP are likely to show poor compliance. Notably, compliance is closely correlated with efficacy and prognosis. In this article, we discussed one case of OP treatment by taking alendronate incontinuously for 20 years. It can be observed that the curative effect and prognosis varied with the treatment compliance. At the same time, a literature review was conducted to gain more insights into OP diagnosis, therapy and treatment course.

\section{Case Report}

She, female, eighty-year-old, received medical examination in our hospital because of pain in her lower back in 20 years ago, and was diagnosed with Primary Osteoporosis with low BMD in the $2-4$ lumbar spines ( $\mathrm{T}$-score $=-3.93, \mathrm{BMD}=$ $0.643 \mathrm{mg} / \mathrm{m}^{2}$, as shown in Table 1) by dual energy X-ray absorptiometry (DXA) while secondary factors were excluded. The patient was prescribed Alendronate (70 mg weekly), Caltrate ( $600 \mathrm{mg}$ daily) and calcitriol ( $0.25 \mathrm{ug}$ daily) for 2 years. In the examination of the $2-4$ lumbar spines after 2 years, she found her BMD increased (T-score $=-3.54, \mathrm{BMD}=0.683 \mathrm{mg} / \mathrm{m}^{2}$, as shown in Table 1 ) and decided to discontinue the medication without consulting her doctor. Eleven years ago, she took another BMD reexamination and getting a decreased BMD in L2-4 (T-score $=-4.68, \mathrm{BMD}=0.434 \mathrm{mg} / \mathrm{m}^{2}$, as shown in Table 1 ); however, she didn't follow the doctor's advice. Nine years ago, the patient slipped and fell on a rainy day, which caused a fracture in her right wrist. After receiving treatment in the Orthopedics Department, she came to visit our department and we suggested her to follow the regulatory treatment with Alendronate, Caltrate and calcitriol based on elementary health education for the patient. Her BMD in L2-4 increased gradually according to the examination results taken respectively in seven $\left(\mathrm{T}\right.$-score $=-2.89, \mathrm{BMD}=0.748 \mathrm{mg} / \mathrm{m}^{2}$, as shown in Table 1 ), five $(\mathrm{T}$-score $=-2.1, \mathrm{BMD}=0.863 \mathrm{mg} / \mathrm{m}^{2}$, as shown in Table 1 ), and three years ago (T-score $=-1.5, \mathrm{BMD}=0.954 \mathrm{mg} / \mathrm{m}^{2}$, as shown in Table 1 ), with the type I procollagen-N-propeptide (P1NP) of $14.4 \mathrm{ng} / \mathrm{ml}, \beta$-Cross of $0.2 \mathrm{ng} / \mathrm{ml}$ and N-MTD of $12.16 \mathrm{ng} / \mathrm{ml}$ (as shown in Table 2) in the examination. Three years ago, the patient slumped down onto the ground with her hips on another rainy day and no fracture was incurred by the accident. Considering the marked increase in BMD, the patient's compliance for the regulatory medication of Fosamax for 6 years, 
Table 1. The bone mineral density (BMD) at different periods.

\begin{tabular}{cccccccc}
\hline & $\begin{array}{c}11, \text { Nov, } \\
1998\end{array}$ & $\begin{array}{c}1, \mathrm{Dec}, \\
2000\end{array}$ & $\begin{array}{c}20, \mathrm{Mar}, \\
2007\end{array}$ & $\begin{array}{c}31, \mathrm{Aug}, \\
2011\end{array}$ & $\begin{array}{c}24, \mathrm{Dec}, \\
2013\end{array}$ & $\begin{array}{c}29, \mathrm{Apr}, \\
2015\end{array}$ & $\begin{array}{c}19, \mathrm{Dec} \\
2016\end{array}$ \\
\hline $\begin{array}{c}\text { lumbar } \\
\text { spine BMD } \\
\text { lumbar }\end{array}$ & 0.643 & 0.683 & 0.434 & 0.748 & 0.863 & 0.908 & 0.894 \\
$\begin{array}{c}\text { spine BMD } \\
\text { T score }\end{array}$ & -3.93 & -3.54 & -4.68 & -2.89 & -2.1 & -1.5 & -2.0 \\
$\begin{array}{c}\text { Hip BMD } \\
\begin{array}{c}\text { Hip BMD T } \\
\text { score }\end{array}\end{array}$ & 0.578 & 0.584 & 0.684 & 0.680 & 0.672 & 0.750 & 0.764 \\
\hline
\end{tabular}

Note: the unit of BMD is $\mathrm{mg} / \mathrm{m}^{2}$.

Table 2. The bone metabolic markers (BTM) at different periods.

\begin{tabular}{rccc}
\hline & PINP (ng/ml) & B-Cross (ng/ml) & N-MID (ng/ml) \\
\hline 14, May, 2015 & 14.44 & 0.2 & 12.16 \\
19, Dec, 2016 & 16.93 & 0.107 & 12.56 \\
12, Mar, 2018 & 21.11 & 0.21 & 20.66 \\
\hline
\end{tabular}

the absence of any new-onset fractures or risk factors and the markers for bone metabolism indicating an inhibitory state of bone resorption, her attending physician approved the discontinuation of Fosamax and has conducted regular follow-ups until now. In the withdrawal period, no fracture occurred to the patient even though she took a tumble on a bus because of an emergency brake during the first month of withdrawal. The patient received another reexamination of her BMD two years ago (as shown in Table 2) and was informed of a slight decrease in BMD, despite of which, the markers for bone metabolism still suggested an inhibition against resorption. Therefore, the patient was approved to continue her drug holiday. And the patient received reexamination of her BMD two months ago (as shown in Table 2), as the result was still suggested an inhibition against resorption, the patient also continued her drug holiday.

Previous medical history: Menopause at the age of 52, diabetes, hypertension.

Physical examination: Former height of $154 \mathrm{~cm}$, present height of $150 \mathrm{~cm}$, shorter than before.

The patient followed relevant instructions carefully when taking alendronate and thus she suffered no adverse gastrointestinal effects. The authors obtained a deeper insight into OP and its treatment when summarizing the whole treatment process.

\section{Discussion}

\subsection{The Present Status of Osteoporosis}

With the sound development of the medical treatment and healthcare sector, the average life expectancy extends while the aging population in the society continues to increase, accompanied by a significantly higher prevalence of senile 
diseases. Almost every individual at an advanced age is exposed to the risk of having OP at a varied level and with different symptoms. In 2007, there were 90 million patients diagnosed with OP in China, equal to $7.1 \%$ of the national population, making China the most severely stricken country across the world. Although the number of OP cases dramatically increases with the aging society, many patients are asymptomatic and they do not seek medical attention until fractures occur [2]. Height loss and humpback caused by OP will tarnish one's personal image severely, exerting adverse impacts on the elderly patients physically and psychologically, and even leading to depression [3]. Moreover, OP-related fragility fractures are considered a heavy burden and severe trauma to patients and their families because they substantially raise the possibility of disability and fatality. It is found in an epidemiological study on the proportion of fractures as a consequence of bone fragility to trauma in elderly people that the occurrence of $\mathrm{OP}$ is associated with the substantial increase in fragility fractures in elderly people [4]. It is reported that a fracture case occurs every three seconds worldwide [5]. According to the estimate results in 2015, China is anticipated to spend RMB 132 billion and RMB 163 billion on the treatment for the population with osteoporotic fractures by 2035 and 2050, respectively [6]. A study indicates that OP treatment can significantly increase a patient's BMD and reduce the risk of fractures [7]. However, considering the low rates of OP diagnosis and treatment, OP treatment and management has become a major issue in the public health sector [8]. To lead a happy and healthy life in their golden years, patients should heighten their awareness of the disease, make early diagnosis and treatment, improve their BMD levels and lower the risk of fractures.

\subsection{Risk Factor for Osteoporosis}

The risk factors for OP not only consist of unhealthy lifestyles, but also include chronic diseases related to the endocrine system, blood, gastrointestinal system and rheumatic immune system (e.g., diabetes, coronary artery disease, hypertension, hyperlipemia, Parkinson's disease) and medications for chronic diseases (e.g., glucocorticoid (GC), antiviral drugs, proton pump inhibitor) [9]. To achieve early diagnosis, on one hand, the IOF One-Minute Osteoporosis Risk Test and the Osteoporosis Self-Assessment Tool for Asians (OSTA) can be introduced to communities so that people can discover their risk of OP and seek medical advice promptly in case of any abnormalities, instead of complaints of lumbar vertebrae pain and even fractures [10]; on the other hand, physicians who focus on other relevant diseases should obtain an overall knowledge of OP and suggest their patients with relevant diseases or receiving medications that are considered to be risk factors for osteoporosis to seek medical attention in the department of OP as early as possible.

\subsection{The Treatment of Osteoporosis}

With regard to medications for OP, a large number of patients incline to calcium and Vitamin D supplements. In fact, it was reported that calcium and Vitamin D 
supplements cannot inhibit OP efficiently [11]. Appropriate therapy should comprise calcium and Vitamin D supplements and anti-osteoporosis drugs. At present, anti-osteoporosis drugs are mainly divided into bone resorption inhibitors and bone formation accelerators [12]. The most extensively used anti-bone resorptive agents are bisphosphonates while the miscellaneous bone resorption inhibitors include calcitonin, estrogen and selective estrogen receptor modulators (SERMs). Bisphosphonates are a class of drugs that inhibit bone resorption, increase BMD and prevent the loss of bone density. They can reduce the risk of fractures by $40 \%$ to $70 \%$ [13].

Alendronate is presently one of the most popular first-line bisphosphonates in China. It has a very high affinity for calcium hydroxyapatite, allowing for rapid and specific skeletal targeting. As a result, it inhibits osteoclasts from destroying and digesting bone, thereby slowing bone loss and reducing the OP symptoms [14]. From a randomized, double-blind, placebo-controlled extension trial conducted in Hong Kong, which involves 100 osteoporotic Chinese women having undergone menopause, it can be seen that the BMD of the alendronate group is increased to a markedly higher level than the placebo group and the difference shows statistical significance. Meanwhile, there is no significant difference between the two groups in adverse gastrointestinal symptoms [15]. In Chapurlat $\mathrm{RD}$ et al., the analysis on the correlations between alendronate treatment and the risk of fractures suggests that patients who "gained" BMD during treatment with alendronate have a considerable reduction in the risk of fractures [16].

Presently, our patients with OP are recommended a weekly dosage of $70 \mathrm{mg}$ alendronate (Fosamax) on an empty stomach. On the convenient medication, they need not to take medicine every day, which improves their compliance but fails to produce an immediate effect. The patients are suggested to adhere to the treatment with alendronate for three to five years while those who are at an advanced age and have a high risk of fractures due to considerably low BMD in their hips are recommended a longer treatment course. For those who have completed their courses of treatment, if the BMD reexamination results after a year or relevant bone turnover marker measurements after two to three years indicate a low risk of fractures, they may continue to monitor their BMD or bone turnover markers [17]. In this case study, the patient had undergone treatment with alendronate for two years during the initial stage of the disease and her BMD was therefore increased. However, she withdrew from the treatment and discontinued the medication without consulting her attending physician, leading to a continuous decrease in BMD. What's worse, she suffered a fragility fracture during the withdrawal period, which increased her pain and medical burden. It should be noted that some patients die of secondary infections and other complications when they are hospitalized for trauma and fractures. Fortunately, the patient recovered well and promptly improved her compliance. Because the patient accepted her physician's recommendation and received regulatory treatment, significant curative effect was achieved and the BMD was in- 
creased. Later, she fell twice on different occasions but did not have any fractures, which is largely contributed by her compliance. According to a retrospective study that assesses the association of the risk of refracture with the compliance and persistence with bisphosphonate therapy based on 56,036 cases in Taiwan, treatment course is negatively correlated with compliance. Specifically, during the research, the patient compliance significantly declined as the treatment went on; only around $30 \%$ of the 56,036 patients adhered to the therapy at one year. Besides, it is reported that a high level of compliance can substantially lower the refracture risk whereas poor compliance implies an increase in the refracture risk [18].

\subsection{Osteoporosis Treatment Compliance}

Despite all that, a patient's compliance with OP treatment is affected by numerous factors. There is a domestic study of the compliance in elderly males with OP who were treated with oral alendronate and the findings indicate that the following factors may lead to poor compliance with alendronate therapy: 1) the patient is not reminded to take his/her medicine; 2) more than seven types of drugs are prescribed; 3 ) the patient fails to be aware of the long-term efficacy; 4) the patient worries about relevant side effects; meanwhile, it is observed that bone pain can improve patient compliance [19]. Another study discusses the association of gastrointestinal events with osteoporosis treatment initiation and treatment compliance in Germany, which suggests that in German women newly diagnosed with osteoporosis, gastrointestinal events reduce the likelihood of receiving treatment and are associated with the choice of treatment. For those initiating oral bisphosphonates, post-treatment gastrointestinal events are associated with the decline in patient compliance [20]. In addition, a patient's awareness of the disease and the therapeutic burden brought by the treatment are also related to his/her compliance. At present, $\mathrm{OP}$ is not deemed as a chronic disease covered by medical insurance. However, a patient diagnosed with OP, on average, has to spend over RMB 3000 on anti-osteoporosis drugs every year; this becomes an extremely heavy burden for those at an advanced age since they are likely to have complicating diseases and thereby considerably lowers their compliance. The weight of $\mathrm{OP}$ in the medical system is another factor that influences patient compliance. For such a disease requiring a relatively long treatment course and producing slow curative effect, the medical insurance system should be improved to relieve the patients' burden, and more importantly, health education and publicity should be carried out to raise the awareness of OP so that the patients can combat the disease in proper ways.

Based on the characteristics of the elderly population, it is of great importance to raise the compliance level among elderly patients. Considering that they are likely to miss several doses during the treatment course, it is necessary for the patients to receive health education, like learning and teaching their families to set reminders to avoid missing any doses. To prevent adverse reactions caused 
by high-risk drugs, physicians should gain thorough knowledge of each patient's medical history before treatment. Adverse gastrointestinal events such as nausea, vomiting, esophageal erosions, gastric and duodenal ulcer, are common reactions brought by alendronate therapy. If a physician is clearly aware that his/her patient has a history of gastrointestinal diseases before treatment, e.g., gastritis, gastric ulcer, erosive esophagitis, alendronate should be replaced with other appropriate bone resorption inhibitors. Likewise, if a patient shows menopausal signs and symptoms, raloxifene should be avoided. Oral alendronate can lead to adverse gastrointestinal symptoms; moreover, oral administration indicates low bioavailability. Patients should remain at rest or keep walking for over $30 \mathrm{mi}$ nutes after they have taken alendronate orally. Presently, researchers are working on the development of a novel transdermal delivery system of alendronate using a new type of hydrophilic patch and experimental animals and dissolving microneedle arrays [21]. The new medication is expected to produce higher curative effect and patient compliance. In fact, the patient in this case study showed no adverse gastrointestinal reactions during the long-term use of alendronate, which appears to be closely related to her strict compliance with the given precautions of drug use. Before prescribing alendronate, physicians should confirm with patients whether they have a history of gastrointestinal diseases and request them to follow relevant instructions strictly to improve compliance and prevent the occurrence of adverse drug reactions.

\subsection{Prevention and Treatment of Fragility Fracture}

The ultimate goal of OP treatment is to prevent fragility fractures. Except for increasing bone density and strength based on early diagnosis and complete treatment of OP, fall prevention also plays a prominent role in preventing osteoporotic fractures. Affected by functional decline, failing eyesight and basic diseases, elderly people are likely to show poor physical coordination and suffer accidental falls, which in most cases is the direct cause of fragility fractures [22]. As shown in Dr. Yu Pulin's study on accidental falls among elderly people of urban community dwellers in Beijing, $18 \%$ of the elderly people have experienced accidental falls [23]. In the global longitudinal study of osteoporosis in women (GLOW), 60,393 postmenopausal women diagnosed with OP were recruited and 4122 incident fractures were reported during the first three years of follow-up, including $8 \%$ lumbar vertebrae and $6 \%$ hip fractures. Fractures were primarily and directly caused by falls [24]. Therefore, fall prevention is an essential strategy to prevent fragility fractures in patients with OP. As to the patient in this case study, her first fracture was caused by an accidental fall nine years ago. Fortunately, she had received standard treatment after the fracture and the following reexamination suggested a significant increase in the BMD. During the treatment course, the patient suffered another two falls that did not lead to fractures.

For those who already have fractures, effective intervention should be made 
promptly to prevent refracture. There is a study that analyzes the risk factors for refracture based on $22 \mathrm{OP}$ cases and indicates that patients with initial osteoporotic fractures have a substantially high risk of refracture while the second fracture is reported to occur 3.7 years after the first on average, which means there is adequate time between the first and second fractures for interventions to reduce the risk of refracture, especially for elderly women with a vertebral or hip fracture. Effective interventions include motor functional rehabilitation and fall prevention training [25].

\subsection{The Application of Bone Metabolic Markers}

Metabolic activity brings about dynamic changes and constant remodeling of bone tissue while OP is a result of imbalanced bone remodeling caused by an increase in osteoclasts and metabolic activity. Metabolic products, namely bone metabolic markers, which are released during bone remodeling, can be detected in blood and urine. Bone metabolic markers are mainly divided into bone formation and resorption markers. Type I procollagen-N-propeptide (P1NP) is a bone formation marker, which reflects the changes of newly synthesized type I collagen. $\beta$-Crosslaps $(\beta$-CTx), as bone resorption markers, are degradation products of type I collagen C-telopeptides. The bone turnover marker $\mathrm{N}$-terminal midfragment osteocalcin (N-MID OC) is a bone-specific, calcium binding protein released by bone formation and resorption. $\beta$-CTx, P1NP and N-MID OC are three bone markers recommended by the International Osteoporosis Foundation (IOF) [26]. Despite the fact that these bone metabolic markers cannot be applied to clinical diagnosis independently, they are effective indicators for the evaluation of secondary OP. To be specific, if these bone metabolic markers in a patient increase abnormally, the patient may have secondary OP and requires further clinical diagnosis to identify secondary factors. Also, bone metabolic markers can be used for bone quality and fracture risk evaluation. Compared to BMD, they are more efficient in revealing the action of drugs through dynamic monitoring and improving patient compliance. Apart from this, they are free of X-ray radiation [27]. Bone metabolic markers are especially useful for patients suffering bone mass loss. If the bone metabolic markers in a patient suggest a high bone turnover rate, there is a high risk of substantial bone mass loss, OP and fractures. In this case, early intervention should be made to reduce the likelihood of OP and even fractures. Bone resorption inhibitors can be used to lower bone metabolic indicators and the results are detectable within a short period (about one to several months). On comparison, BMD changes very slowly, which is imperceptible in the first six months or even longer during the treatment course. Therefore, bone metabolic markers are more useful than BMD in clinical evaluation of drug efficacy. Presently, our hospital provides patients with examinations of relevant bone metabolic markers. Taking the patient in this case study for example, in the later stage of the treatment course, she underwent highly individualized examinations combined with bone metabolic in- 
dicators, and the results showed that she was free from new-onset fractures and abnormal changes in BMD and bone metabolism. Based on the examination results, the patient was approved to continue her drug holiday.

\section{Conclusion}

To summarize, OP cases require early diagnosis and intervention, an adequate treatment course and fall prevention to minimize the risk of fragility fractures and improve the quality of life of elderly patients. For those who already have fragility fractures, they should adhere to anti-osteoporosis treatment to prevent the occurrence of refracture. Bone metabolic markers are useful indicators for clinical diagnosis of OP and dynamic curative effect evaluation.

\section{References}

[1] Huang, S., Lin, H., Zhu, X., et al. (2014) Zoledronic Acid Increases Bone Mineral Density and Improves Health-Related Quality of Life over Two Years of Treatment in Chinese Women with Postmenopausal Osteoporosis. Endokrynologia Polska, 65, 96-104. https://doi.org/10.5603/EP.2014.0014

[2] Assessment S C O H. (2003) Osteoporosis-Prevention, Diagnosis and Treatment: A Systematic Review. Swedish Council on Health Technology Assessment (SBU), Stockholm.

[3] Robbins, J., Hirsch, C., Whitmer, R., et al. (2001) The Association of Bone Mineral Density and Depression in an Older Population. Journal of the American Geriatrics Society, 49, 732-736. https://doi.org/10.1046/j.1532-5415.2001.49149.x

[4] Johansen, A., Harding, K., Evans, R., et al. (1999) Trauma inat proportion of fractures Are a Consequence of Bone Fragility? Archives of Gerontology and Geriatrics, 29, 215-221. https://doi.org/10.1016/S0167-4943(99)00034-5

[5] Johnell, O. and Kanis, J.A. (2006) An Estimate of the Worldwide Prevalence and Disability Associated with Osteoporotic Fractures. Osteoporosis International, 17, 1726-1733. https://doi.org/10.1007/s00198-006-0172-4

[6] Liu, L.M. (2017) An Interpretation on Guidelines for Diagnosis and Treatment of Osteoporotic Fractures and a Guide to Diagnosis and Treatment of Primary Osteoporosis. Beijing Medical Journal, 39, 180-182.

[7] Siris, E.S., Harris, S.T., Rosen, C.J., et al. (2006) Adherence to Bisphosphonate Therapy and Fracture Rates in Osteoporotic Women: Relationship to Vertebral and Nonvertebral Fractures from 2 US Claims Databases. Mayo Clinic Proceedings, 81, 1013-1022. https://doi.org/10.4065/81.8.1013

[8] Gauthier, K., Bai, A., Perras, C., et al. (2012) Denosumab, Raloxifene, and Zoledronic Acid for the Treatment of Postmenopausal Osteoporosis: Clinical Effectiveness and Harms. Ottawa (ON): Canadian Agency for Drugs and Technologies in Health.

[9] Cosman, F., de Beur, S.J., LeBoff, M.S., et al. (2014) Clinician’s Guide to Prevention and Treatment of Osteoporosis. Osteoporosis International, 25, 2359-2381. https://doi.org/10.1007/s00198-014-2794-2

[10] Koh, L.K., Sedrine, W.B., Torralba, T.P., et al. (2001) A Simple Tool to Identify Asian Women at Increased Risk of Osteoporosis. Osteoporosis International, 12, 699-705. https://doi.org/10.1007/s001980170070

[11] Reid, I.R., Bristow, S.M. and Bolland, M.J. (2015) Calcium Supplements: Benefits 
and Risks. Journal of Internal Medicine, 278, 354-368. https://doi.org/10.1111/joim.12394

[12] Chinese Society of Osteoporosis and Bone Mineral Research, Chinese Medical Association (2017) A Guide to Diagnosis and Treatment of Primary Osteoporosis (2017 Edition). Chinese Journal of Endocrinology and Metabolism, 33, 890-913.

[13] Arabmotlagh, M., Rittmeister, M. and Hennigs, T. (2006) Alendronate Prevents Femoral Periprosthetic Bone Loss Following Total Hip Arthroplasty: Prospective Randomized Double-Blind Study. Journal of Orthopaedic Research, 24, 1336-1341. https://doi.org/10.1002/jor.20162

[14] Hassler, N., Gamsjaeger, S., Hofstetter, B., et al. (2015) Effects of Long-Term Alendronate Treatment on Postmenopausal Osteoporosis Bone Material Properties. Os teoporosis International, 26, 339-352. https://doi.org/10.1007/s00198-014-2929-5

[15] Lau, E.M., Woo, J., Chan, Y.H., et al. (2000) Alendronate Prevents Bone Loss in Chinese Women with Osteoporosis. Bone, 27, 677-680. https://doi.org/10.1016/S8756-3282(00)00378-1

[16] Chapurlat, R.D., Palermo, L., Ramsay, P., et al. (2005) Risk of Fracture among Women Who Lose Bone Density during Treatment with Alendronate. The Fracture Intervention Trial. Osteoporosis International, 16, 842-848. https://doi.org/10.1007/s00198-004-1770-7

[17] Gourlay, M.L. and Ensrud, K.E. (2014) Bone Density and Bone Turnover Marker Monitoring after Discontinuation of Alendronate Therapy: An Evidence-Based Decision to Do Less. JAMA Internal Medicine, 174, 1134-1135. https://doi.org/10.1001/jamainternmed.2014.162

[18] Soong, Y.K., Tsai, K.S., Huang, H.Y., et al. (2013) Risk of Refracture Associated with Compliance and Persistence with Bisphosphonate Therapy in Taiwan. Osteoporosis International, 24, 511-521. https://doi.org/10.1007/s00198-012-1984-Z

[19] Jiang, Y., Pi, H.Y., Yan, Y.F., et al. (2013) The Compliance in Elderly Male with Osteoporosis Treated with Oral Alendronate. Chinese Journal of Internal Medicine, 52, 387-389.

[20] Modi, A., Fan, C.S., Tang, J., et al. (2016) Association of Gastrointestinal Events with Osteoporosis Treatment Initiation and Treatment Compliance in Germany: An Observational Study. Bone Reports, 5, 208-213. https://doi.org/10.1016/j.bonr.2016.06.001

[21] Katsumi, H. (2014) Development of a Novel Transdermal Delivery System of Alendronate, a Nitrogen Containing Bisphosphonate, Using a New Type of Hydrophilic Patch and Dissolving Microneedle Arrays. Yakugaku Zasshi, 134, 427-431. https://doi.org/10.1248/yakushi.13-00239

[22] Weir, E. and Culmer, L. (2004) Fall Prevention in the Elderly Population. Canadian Medical Association Journal, 171, 724. https://doi.org/10.1503/cmaj.1041381

[23] Yu, P.L. (2010) Epidemiological Characteristics of Falls and Its Prevention Strategies among Elderly People of Urban Community Dwellers in Beijing. Epidemiology and Health Statistics of Central South University.

[24] Costa, A.G., Wyman, A., Siris, E.S., et al. (2013) When, Where and How Osteoporosis-Associated Fractures Occur: An Analysis from the Global Longitudinal Study of Osteoporosis in Women (GLOW). PLoS ONE, 8, e83306. https://doi.org/10.1371/journal.pone.0083306

[25] Ruan, W.D., Wang, P., Ma, X.L., et al. (2011) Analysis on the Risk Factors of Second Fracture in Osteoporosis-Related Fractures. Chinese Journal of Traumatology, 14, 74-78. 
[26] Nishizawa, Y., Ohta, H., Miura, M., et al. (2013) Guidelines for the Use of Bone Metabolic Markers in the Diagnosis and Treatment of Osteoporosis (2012 Edition). Journal of Bone and Mineral Metabolism, 31, 1-15. https://doi.org/10.1007/s00774-012-0392-y

[27] Shetty, S., Kapoor, N., Bondu, J.D., et al. (2016) Bone Turnover Markers: Emerging Tool in the Management of Osteoporosis. Indian Journal of Endocrinology and Metabolism, 20, 846-852. https://doi.org/10.4103/2230-8210.192914 